\title{
PARTICLE FILTER FOR UNDERWATER TERRAIN NAVIGATION
}

\author{
Rickard Karlsson and Fredrik Gustafsson \\ Control and Communication \\ Linköping University \\ SE-58183 Linköping, Sweden \\ E-mail: $\{r i c k a r d$, fredrik\}@isy.liu.se
}

\begin{abstract}
In an earlier contribution we proposed a particle filter for underwater (UW) navigation, and applied it to an experimental trajectory. Here we focus on performance improvements and analysis. First, the Cramér Rao lower bound (CRLB) along the experimental trajectory is computed, which is only slightly lower than the particle filter estimate after initial transients. Simple rule of thumbs for how performance depends on the map and sensor quality are derived. Second, a more realistic five state model is proposed, and Rao-Blackwellization is applied to decrease computational complexity. Monte-Carlo simulations on the map demonstrate a performance comparable to the CRLB.
\end{abstract}

\section{THE NAVIGATION SYSTEM}

In [2], we applied the particle filter on the following model for an underwater (UW) vessel.

Model I. Position model where $x_{t} \in \mathbb{R}^{2}$ is the horizontal position state vector.

$$
\begin{aligned}
x_{t+1} & =x_{t}+u_{t}+\mathrm{w}_{t}, \\
y_{t} & =h\left(x_{t}\right)+e_{t} .
\end{aligned}
$$

Here $y_{t}$ is the measured depth, $u_{t}$ is the INS corrections and $\mathrm{w}_{t}$ the process noise due to drift. We assumed that the velocity vector $u_{t}$ is measured or that, for instance, rudder angle and propeller speed can be converted to a velocity vector. We will here re-visit the Cramér-Rao lower bound (CRLB) calculations in [2], and apply it to an experimental trajectory and map from [2,9]. A more realistic model, often used in aircraft traffic control, is based on the co-ordinated turn assumption (CT).

Model II. CT-model with Cartesian position (x, y), orientation $(\varphi)$ and yaw rate $(\omega)$ and velocity $(v)$ as state variables

$$
x_{t}=\left(\begin{array}{lllll}
\mathrm{x}(t) & \mathrm{y}(t) & \varphi(t) & \omega(t) & v(t)
\end{array}\right)^{T} .
$$

The continuous-time CT-model is discretized assuming constant noise values during the sample period as in [4, p.317]. The discretization of the unknown noise signal can be done

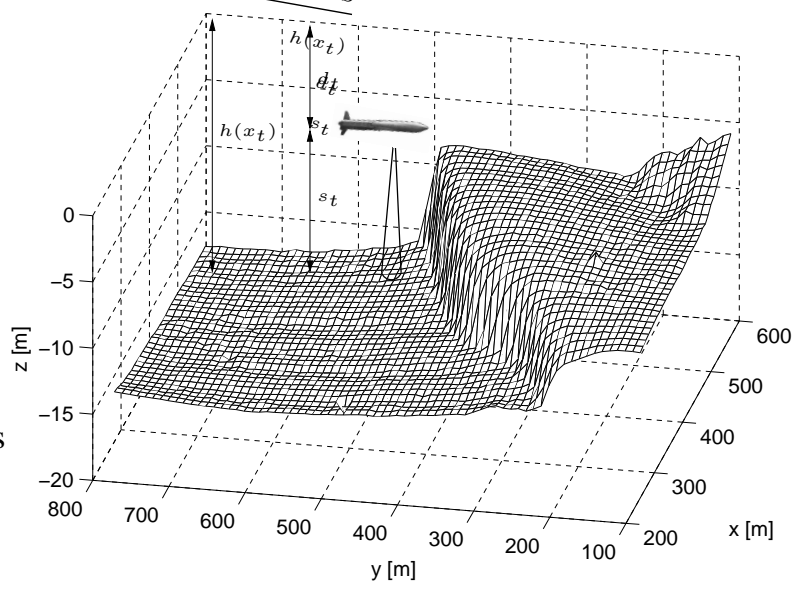

Fig. 1. Underwater navigation using sonar depth measurements and a true UW-terrain data base.

in several ways, but to simplify the model we assume that it is white noise whose influence during one sample is $T Q$ as discussed in [4].

$$
x_{t+1}=\left(\begin{array}{c}
\mathrm{x}_{t}+\frac{2 v_{t}}{\omega_{t}} \sin \left(\frac{\omega_{t} T}{2}\right) \cos \left(\varphi_{t}+\frac{\omega_{t} T}{2}\right) \\
\mathrm{y}_{t}+\frac{2 v_{t}}{\omega_{t}} \sin \left(\frac{\omega_{t} T}{2}\right) \sin \left(\varphi_{t}+\frac{\omega_{t} T}{2}\right) \\
\varphi_{t}+\omega_{t} T \\
\omega_{t}+u_{1}+\mathrm{w}_{1} \\
v_{t}+u_{2}+\mathrm{w}_{2}
\end{array}\right) .
$$

Here the rudder angle and propeller speed are the inputs, which is a more realistic assumption than model $\mathrm{I}$, and that the yaw rate and velocity are smooth highly correlated signals (integrated white noise here). Using a higher number of states is prohibitive for an efficient real-time implementation, so Rao-Blackwellization is applied for that case. The advantage is that more states for sensor and actuator offsets, more sensors like compass, GPS (when available for UW vessels), accelerometers etc., as well as disturbance states as current drifts are easily incorporated, without substantially increasing the complexity. In Fig. 1, the system is described. The sonar measurement is denoted $s_{t}$, the vessel's depth, $d_{t}$, and the database depth in location $x_{t}$, is $h\left(x_{t}\right)$. 


\section{PARTICLE FILTER}

To solve the non-tractable Bayesian UW-navigation problem in an on-line application without using linearization or Gaussian assumptions, sequential Monte Carlo methods, or particle filters, could be used. Here only a brief description of the theory is given. For more details we refer to $[1,3,5,6]$. Consider

$$
\begin{aligned}
x_{t+1} & =f\left(x_{t}, u_{t}, \mathrm{w}_{t}\right), \\
y_{t} & =h\left(x_{t}\right)+e_{t},
\end{aligned}
$$

where $x_{t} \in \mathbb{R}^{n}$ denotes the state of the system, $u_{t}$ the input signal and $y_{t}$ the observation at time $t$. The process noise $\mathrm{w}_{t}$ and measurement noise $e_{t}$ are assumed independent with densities $p_{\mathrm{w}_{t}}$ and $p_{e_{t}}$ respectively. Let $\mathbb{Y}_{t}=\left\{y_{i}\right\}_{i=1}^{t}$ be the set of observations until present time. The particle filter method provides an approximative Bayesian solution to

$$
\begin{aligned}
p\left(x_{t+1} \mid \mathbb{Y}_{t}\right) & =\int_{\mathbb{R}^{n}} p\left(x_{t+1} \mid x_{t}\right) p\left(x_{t} \mid \mathbb{Y}_{t}\right) d x_{t} \\
p\left(x_{t} \mid \mathbb{Y}_{t}\right) & =\frac{p\left(y_{t} \mid x_{t}\right) p\left(x_{t} \mid \mathbb{Y}_{t-1}\right)}{p\left(y_{t} \mid \mathbb{Y}_{t-1}\right)}
\end{aligned}
$$

by approximating the probability density $p\left(x_{t} \mid \mathbb{Y}_{t}\right)$ by a large set of $N$ particles $\left\{x_{t}^{(i)}\right\}_{i=1}^{N}$, where each particle has an assigned relative weight, $\gamma_{t}^{(i)}$, such that all weights sum to unity. The location and weight of each particle reflect the value of the density in the region of the state space. The likelihood $p\left(y_{t} \mid x_{t}\right)$ is calculated from (4) yielding

$$
\gamma_{t}=p\left(y_{t} \mid x_{t}\right)=p_{e_{t}}\left(y_{t}-h\left(x_{t}\right)\right) .
$$

By introducing a resampling step as in [5] problems with divergence can be handled. This is referred to as sampling importance resampling (SIR), and is summarized in Alg 1.

\section{Sampling Importance Resampling (SIR)}

1. Generate $N$ samples $\left\{x_{0}^{(i)}\right\}_{i=1}^{N}$ from $p\left(x_{0}\right)$.

2. Compute $\gamma_{t}^{(i)}=p_{e}\left(y_{t} \mid x_{t}^{(i)}\right)$ and normalize, i.e., $\bar{\gamma}_{t}^{(i)}=\gamma_{t}^{(i)} / \sum_{j=1}^{N} \gamma_{t}^{(j)}, i=1, \ldots, N$.

3. Generate a new set $\left\{x_{t}^{(i \star)}\right\}_{i=1}^{N}$ by resampling with replacement $N$ times from $\left\{x_{t}^{(i)}\right\}_{i=1}^{N}$, with probability $\bar{\gamma}_{t}^{(j)}=\operatorname{Pr}\left\{x_{t}^{(i \star)}=x_{t}^{(j)}\right\}$

4. Prediction: $x_{t+1}^{(i)}=f\left(x_{t}^{(i \star)}, u_{t}, \mathrm{w}_{t}^{(i)}\right), i=1, \ldots, N$ using different noise realizations.

5. Increase $t$ and iterate to step 2 .

Alg. 1. Sampling Importance Resampling.

If part of the state vector is linear-Gaussian the computational complexity can be reduced in many cases using the Rao-Blackwellizations technique [7, 8]. In principle the posterior density is divided in two parts by conditioning and applying Bayes theorem. Hence, the conditional linearGaussian states are estimated with a Kalman filter (one for each particle, but with the structure below the covariance is the same for all) and the nonlinear states with the particle filter. Partitioning the state vector in (3) as $x_{t}=\left(z_{t}^{T}, m_{t}^{T}\right)^{T}$, where $z_{t}=(\varphi, \omega, v)^{T}$ and $m_{t}=(\mathrm{x}, \mathrm{y})$, the structure with the linear-Gaussian equation (7b) is given by

$$
\begin{aligned}
z_{t+1} & =f\left(z_{t}, m_{t}\right)+\mathrm{w}_{z, t}, \\
m_{t+1} & =A_{t} m_{t}+\mathrm{w}_{m, t}, \\
y_{t} & =h\left(z_{t}\right)+C_{t} m_{t}+e_{t} .
\end{aligned}
$$

The algorithm is summarized in Alg 2.

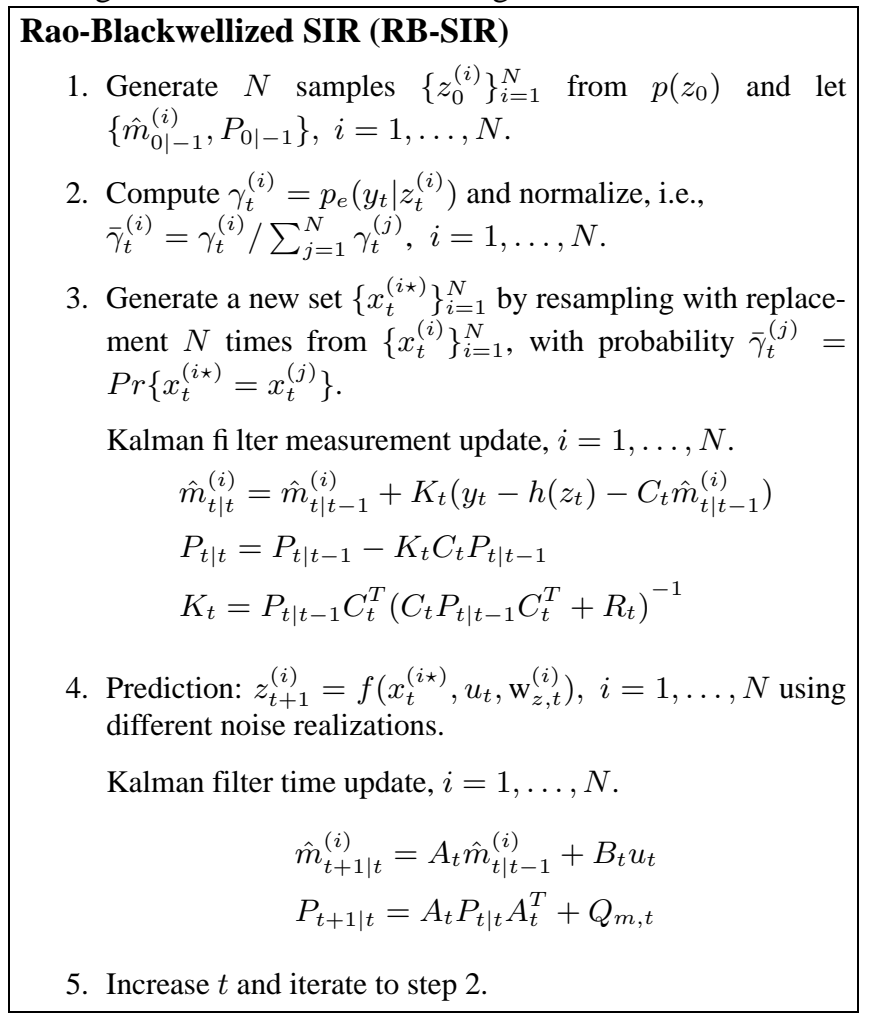

Alg. 2. Rao-Blackwellized SIR (RB-SIR) for eq. (7).

\section{THE CRAMÉR-RAO LOWER BOUND (CRLB)}

The simplified model (model I) consists of position only in order to derive and interpret the CRLB in terms of fundamental noise properties. The observation relation consists of sonar measurements of the depth, where $e_{t}$ is the measurement noise. Using standard notations we consider independent noise sources, with variances $Q_{t}=\mathbb{E}\left\{\mathrm{w}_{t} \mathrm{w}_{t}^{T}\right\}$ and $R_{t}=\mathbb{E}\left\{e_{t} e_{t}^{T}\right\}$. The CRLB for one step prediction with models according to (1) is given in $[1,3]$. We can formulate this as

$$
P_{t+1}=\left(P_{t}^{-1}+\mathbb{E}\left\{\varphi\left(x_{t}\right) R_{t}^{-1} \varphi^{T}\left(x_{t}\right)\right\}\right)^{-1}+Q_{t},
$$


where $P_{t}$ is the covariance matrix for the estimation error, evaluated around the position $x_{t}$ and where

$$
\varphi\left(x_{t}\right)=\left.\nabla_{x} h(x)\right|_{x=x_{t}} .
$$

For scalar measurements, $R_{t}=r$, we have

$$
\begin{aligned}
& P_{t+1}=\left(P_{t}^{-1}+r^{-1} Z_{t}\right)^{-1}+Q_{t}, \\
& Z_{t}=\mathbb{E}\left\{\varphi\left(x_{t}\right) \varphi^{T}\left(x_{t}\right)\right\} .
\end{aligned}
$$

We are interested in the stationary behavior in each position, i.e., $Z_{t}=Z(x)$. The assumption is that we get the global behavior by studying the covariance locally in each position. For stationary systems, $P_{t} \rightarrow \bar{P}(x)$, we have

$$
\begin{aligned}
\bar{P}(x) & =\left(\bar{P}^{-1}(x)+r^{-1} Z(x)\right)^{-1}+Q \\
& =\left(I+\bar{P}(x) r^{-1} Z(x)\right)^{-1} \bar{P}(x)+Q \\
& \approx\left(I-\bar{P}(x) r^{-1} Z(x)\right) \bar{P}(x)+Q .
\end{aligned}
$$

Hence the covariance $\bar{P}$ for the CRLB is given by

$$
\bar{P} r^{-1} Z(x) \bar{P}=Q,
$$

under the assumption that the Taylor expansion is valid, i.e., $\bar{P} r^{-1} Z(x)$ is small. If not the covariance is iterated until convergence. The actual value of $Z$ is given by substituting the expected mean by the sample average in a neighborhood of $x$. It is natural to assume $Q=q \cdot I_{2 \times 2}$. Factorization of the symmetric positive-definite matrix $Z=\Lambda \Lambda^{T}$ using a symmetric matrix square root $\Lambda$ in (13) yields

$$
q \cdot I_{2 \times 2}=r^{-1} \bar{P} \Lambda \Lambda^{T} \bar{P}^{T}=r^{-1} \bar{P} \Lambda(\bar{P} \Lambda)^{T} .
$$

Identifying $\bar{P}=\Lambda^{-1} \sqrt{q r}$, we have $\bar{P}^{2}=\bar{P} \bar{P}^{T}=q r Z^{-1}$. We can directly interpret this relation, for example increased model uncertainty $(q)$ yields higher covariance. Conversely, a better sensor (smaller $r$ ) or higher terrain excitation (larger $Z$ ) reduces $\bar{P}$. By subtracting the process noise the CRLB for estimation is given, which can be compared with the root mean square error (RMSE) from Monte Carlo simulations.

\section{EXPERIMENTS AND SIMULATIONS}

The particle filter is tested on experimental data from an UW-vessel system as well as evaluating Monte Carlo simulations from simulation data. The CRLB calculations from Section 3 are compared to the particle filter RMSE.

CRLB and map generation. In [9] an UW-terrain map was collected using sonar depth measurements and differential GPS. In Fig. 3 (a) the original data is shown. The depth data is here resampled and interpolated to a uniform grid presented in Fig. 1. If the level curves are studied one can see that the terrain is sufficiently varied even in the somewhat flat regions for successful positioning. The CRLB values for each position in the map are given in Fig. 2 with

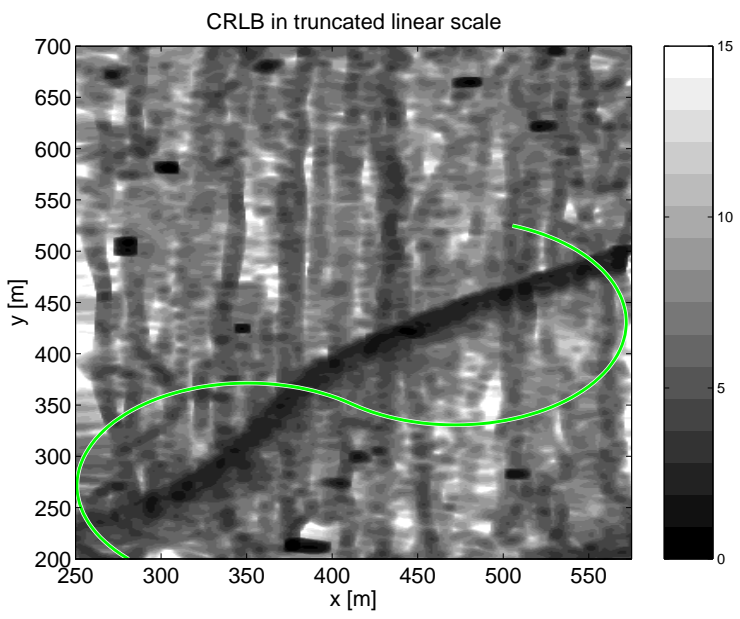

Fig. 2. CRLB $\|\bar{P}\|$ for each position and the trajectory (light grey line) used in the Monte Carlo simulations.

$\|\bar{P}\|=\sqrt{\operatorname{tr}\{\bar{P}\}}$. CRLB values above $15 \mathrm{~m}$ are truncated to enhance the resolution in interesting regions.

Experimental test run. The SIR method is tested on the UW-map and initialized by placing particles uniformly over the entire map. The process and measurement noises are assumed Gaussian with covariances $Q=I_{2 \times 2}$ and $R=0.1$ respectively. The filter uses model $\mathrm{I}$ and is initialized with $N=20000$ particles, but after a few iteration it is reduced to 5000. The depth of the vessel is constant during the experiment. The input signal $u_{t}$ is from the GPS computed position since no true speedometer was present. However, the signal is perturbed to emulate true performance by adding an error of 10 percent with a uniform distribution. In Fig. 3 (a) the data used in the depth map generation is shown, together with the vessels true trajectory. In Fig. 3 (b) the mean value estimate is shown from the particle filter. The original sample rate was $10[\mathrm{~Hz}]$, but data was decimated so the filter was updated with $0.2[\mathrm{~Hz}]$. The model used in the experiment is the one presented in Section 3. Also in Fig. 3 (b) the RMSE from the particle filter is presented together with the CRLB as a function of time. The CRLB is not calculated in regions where the depth data is too sparse to ensure a well defined gradient. In Fig. 3 (b) it is indicated approximately when the vessel is close to the steep UW-slope. Note also that the RMSE is from a true experiment, so only one realization is compared to the ensemble averaged CRLB.

Monte Carlo simulations. In a Monte Carlo simulation the particle filter performance is evaluated using 500 simulations on the true depth map, but with simulated sensors using the trajectory indicated in Fig. 2. Both the SIR and the RB-SIR are evaluated using the RMSE as seen in Fig. 4 using $N=10000$ and $N=2000$ particles respectively. These are reduced by half at sample 20 and 50 . In the 


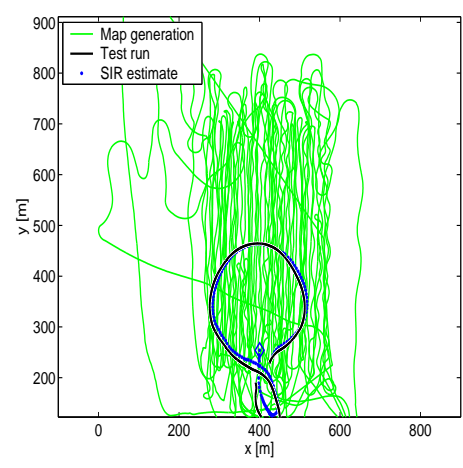

(a) Map generation and test run

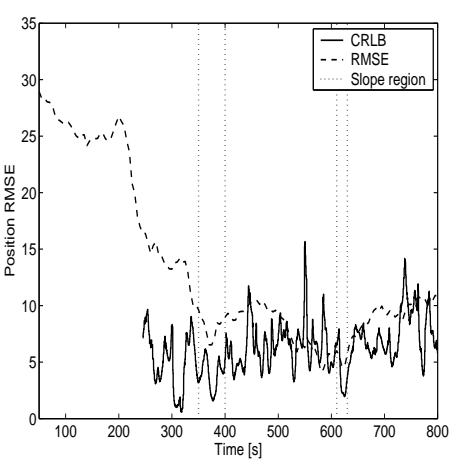

(b) CRLB and RMSE from SIR

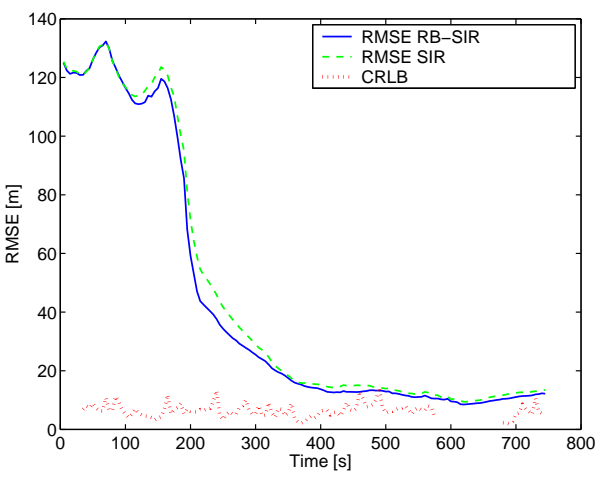

Fig. 4. RMSE for 500 Monte Carlo simulations.
Fig. 3. Experimental test run and map generation trajectory.

RMSE calculation divergent simulations were removed, 8 for SIR and 4 for RB-SIR. RB-SIR thus gives fewer divergences and lower RMSE with a much smaller number of particles. The comutational burden (measured in simulation time) was about $30 \%$ lower for RB-SIR. The cloud is initialized uniformly over the map positions corresponding to a depth close to the initial measurement. Here model II is used with sample time $T=5 \mathrm{~s}$, with measurements of depth $\left(s_{t}\right)$, speed $(v)$ and yaw rate $(\omega)$. We consider disturbances (process noise) in yaw rate $(\omega)$ and speed $(v)$. The process noise $Q$ and measurement noise $R$ are assumed Gaussian with values

$$
Q=\left(\begin{array}{cc}
4 \cdot 10^{-6} & 0 \\
0 & 9 \cdot 10^{-2}
\end{array}\right), R=\left(\begin{array}{ccc}
10^{-2} & 0 & 0 \\
0 & 4 \cdot 10^{-2} & 0 \\
0 & 0 & 10^{-6}
\end{array}\right)
$$

For the RB-SIR method the following matrices are used in the Kalman filter

$$
A=I_{3 \times 3}, C=\left(\begin{array}{ccc}
0 & 0 & 0 \\
0 & 0 & 1 \\
0 & 1 & 0
\end{array}\right) .
$$

To improve tracking performance for this particular model a jittering noise is also added to all components of the state vector.

\section{CONCLUSIONS}

We have addressed performance issues of the particle filter algorithm for UW navigation proposed in [2]. First, CRLB computed on a measured test trajectory shows that the particle filter almost attains the performance bound. Second, a more flexible model was proposed, which in conjunction with Rao-Blackwellization promises a very flexible highperformance real-time algorithm which is easily extended with more states. Monte Carlos simulations are used to verify that the RMSE is close to the CRLB.
The authors would like to thank Saab Bofors Underwater Systems, Björn Johansson, Anna Falkenberg and Tobias Karlsson for providing UW-topological data.

\section{REFERENCES}

[1] N. Bergman, Recursive Bayesian Estimation: Navigation and Tracking Applications, Ph.D. thesis, Linköping University, 1999, Dissertations No. 579.

[2] R. Karlsson, F. Gustafsson, and T. Karlsson, "Particle Filtering and Cramér-Rao Lower Bound for Underwater Navigation," in Proc. IEEE Conf. Acoustics, Speech and Signal Processing (ICASSP), Hong Kong, Apr 2003.

[3] A. Doucet, N. de Freitas, and N. Gordon, Eds., Sequential Monte Carlo Methods in Practice, Springer Verlag, 2001.

[4] F. Gustafsson, Adaptive Filtering and Change Detection, John Wiley \& Sons Ltd, 2000.

[5] N.J. Gordon, D.J. Salmond, and A.F.M. Smith, "A Novel Approach to Nonlinear/non-Gaussian Bayesian State Estimation," in IEE Proceedings on Radar and Signal Processing, 1993, vol. 140, pp. 107-113.

[6] R. Karlsson, Simulation Based Methods for Target Tracking, Licentiate Thesis no. 930, Department of Electrical Engineering, Linköping University, Sweden, Feb 2002.

[7] F. Gustafsson, F. Gunnarsson, N. Bergman, U. Forssell, J. Jansson, R. Karlsson, and P-J Nordlund, "Particle Filters for Positioning, Navigation and Tracking," IEEE Transactions on Signal Processing, Feb 2002.

[8] P-J. Nordlund, Sequential Monte Carlo Filters and Integrated Navigation, Licentiate Thesis no. 945, Dep. of Electrical Engineering, Linköping University, May 2002.

[9] T. Karlsson, "Terrain Aided Underwater Navigation using Bayesian Statistics," Masters Thesis LiTH-ISY-EX-3292, Dep. of Electrical Engineering, Linköping University, 2002. 